\title{
The future of ophthalmology; how will the patient with ophthalmic problems be cared for in 30 years' time?
}

Keywords: blindness, retinitis pigmentosa, genomics, human eye

\section{Opinion}

Who are the ophthalmologists of the future? How would the next generation practice and how would the rapidly growing technology shape their lives, profession and what effect will it create on their patients' experience? The promising role of technology creates motivation and hope. It opens new horizons about the education, practice, and lifestyle of this stunning field and above all, how the patients of ophthalmology would be cared for in the next 30 years! Researchers estimate that $80-85$ percent of our perception, learning, cognition, and activities are mediated through vision, having said that, blindness and vision impairment which are both significant challenges have dropped markedly during the last few decades. The decrease can be attributed to progress in technology, for example in surgical techniques and concerning treating eye infections.

Looking back not many years ago, the significant change in how the patient used to be cared for compared to the current days is undeniable. Starting from surgical techniques and the machines we use, moving to how we now manage long-term conditions that were nearly impossible to be managed in the most optimistic ophthalmologist's mind back in the days. For example, mind-blowing innovations and great efforts are now dedicated to controlling conditions causing significant sight impairment such as AMD or retinitis pigmentosa. Living in the current era where technology and innovations dominate and influence our lives, remarkable, unprecedented advances are taking place. As expected, tiny, well-designed and connected instruments and the accompanying apps make it possible to undertake eye examinations anywhere in the world - making patients the point of care. Internationally, this considerable step would provide ophthalmic service to people in unprivileged areas and people in war zones. Using smartphones and newly built lenses might be the future of out of hospital ophthalmology. One can imagine how using these remote approached would provide a quicker response to some of the most serious ophthalmic emergencies where time is vital in determining the future of the patients' vision and the quality of their life. For instance, it is not imaginary being able to diagnose retinal artery occlusion or an acute angle closure glaucoma using criteria that does not require the patient to waste valuable time in A\&Es. We have all heard about a watch being able to detect a fall or a life-threatening arrhythmia, not far from today, we might see devices and implants to identify ocular emergencies that significantly reduce the long-term complications of not having responded to an ophthalmic emergency in a timely manner. ${ }^{1,2}$

This dramatic change will in no doubt lead to unprecedented improvements in ophthalmic care. It would not be surprising very soon to see the chronic sight-threatening conditions well-monitored. Virtual clinics and technical apps might give a better connection between the patient and the service allowing the targeted condition to be observed and giving a chance for an intervention to take place as
Volume 9 Issue 5 - 2019

Housam Monla-Haidar
Cardiff and Vale University Health Board, UK

Correspondence: Housam Monla-Haidar, Cardiff and Vale University Health Board, UK, Tel 07459199210, +447459199210, ORCID 0000-0002-4353-4I20,

Email housam.haidar@doctors.org.uk

Received: October 17, 2019 | Published: October 23, 2019

soon as needed. A small artificial lens might measure the intraocular pressure, and a retinal implant might be able to assess the level of macular degeneration and who knows, regular clinic appointments to monitor keratoconus might become something from the past. As the currently used OCT completely wiped out the one used ten years ago, in 30 years, new technical innovations would inspire how the patient in ophthalmology is cared for. For example, digital contact lenses transmitting particular information about the body to an outside device sound like science fiction. Yet, it might be a reality soon. For example, Google teamed up with Novartis, to produce digital, multisensor contact lenses which are designed to be able to measure blood sugar levels. Talking about robotics, femtosecond laser surgery for the removal of cataracts is already here. This technology will only become better and more affordable in the future. Genomics may lead to earlier and better treatment of eye-related ailments like diabetic retinopathy and retinitis pigmentosa. The first successful transplant of a biosynthetic cornea occurred in 2010. Since that time, breakthroughs in biotechnology, material science, and 3D printing have accelerated the field and, in the not-too-distant future, biosynthetic corneas could help restore sight to the vast number of people who are currently waiting for a donated human cornea for transplantation. It is only a matter of time before the neurons in the human retina are similarly mapped. When this happens ophthalmologists understanding of photoreceptors, horizontal cells, bipolar cells, amacrine cells, and ganglion cells may lead to a new and deeper understanding of the human eye. Mentioning the rapid change in all medical and surgical specialties makes the difference in how the patient is looked after in ophthalmology promising. In 30 years, with all the above ideas and efforts, the patient in ophthalmology would be looked after more efficiently, ophthalmic emergencies would be dealt with rapidly, chronic diseases are expected to be more controlled and newer diagnostic and surgical devices, facilities and techniques would make the patient's care more satisfying and affordable more than ever.,

\section{Acknowledgments}

None. 


\section{Funding}

Nill.

\section{Conflicts of interest}

Author declares that there is no conflict of interest.

\section{References}

1. Jack Uldrich. The Future of Ophthalmology: 10 Trends Transforming Tomorrow. 2013.
2. Omoniyi Y. Researchers may be able to predict glaucoma risk. Fight for Sight. 2018.

3. Purnima S Patel MD, Jeff H Pettey MD. 4 Ideas that Will Change Ophthalmologyss Future. Annual Meeting: american academy of ophthalmology. 2016.

4. The Medical Futurist. The Future of Vision and Eye Care. 2017. 